\title{
Pharmacokinetic Variability in Pediatrics and Intensive Care: Toward a Personalized Dosing Approach
}

\author{
Amélie Marsot \\ Faculté de Pharmacie-Université de Montréal \\ Received, July 19, 2018; Accepted, September 9, 2018; Published, September 16, 2018.
}

\begin{abstract}
Providing a safe and efficacious drug therapy for large and often heterogeneous populations is a challenging objective in clinical drug development and routine clinical practice. It has been known for years that the optimum dose required for many therapeutic agents among individuals is quite variable. A wide interindividual pharmacokinetic variability was described in clinically relevant populations such as pediatrics and critically ill patients. The aim of this article was to present the main individual factors influencing variability in these two populations and their applications. Growth and development are two specific features of children that are not observed in adults. And critically ill patients have a much higher level of sickness severity that is associated with profound pathophysiological changes. These particular features could lead to difficulties to attain therapeutic targets. Nonlinear mixed effects modeling is a common approach to identify unexplained population variability. This approach is often applied to evaluate and optimize drug therapy in particular populations. Numerous studies have been conducted in these two specific populations to characterize pharmacokinetic parameters and to identify individual factors influencing variability. Size, age and organ function appeared to be the main factors influencing pharmacokinetics in pediatrics. Factors influencing pharmacokinetics in critically ill patients were mainly cardiovascular system, organ dysfunction and organ support. Dosage individualization seems to be a key issue to optimize drug treatment in these specific populations. Clinically utility and safety of a model-based personalized drug therapy has been demonstrated for vancomycin in pediatrics. Many programs were available to optimize drug regimens, especially for antibiotic drugs in critically ill patients. This innovative personalized dosing approach is a promising way to optimize drug therapy in clinically relevant populations, such as pediatrics and critically ill patients.
\end{abstract}

\section{INTRODUCTION}

Providing a safe and efficacious drug therapy for large and often heterogeneous populations is a challenging objective in clinical drug development and routine clinical practice. On the one hand, a therapeutic effect of the drug is desired to be achieved for all patients; on the other hand too high concentrations have to be avoided to reduce adverse events [1,2]. It has been known for years that the optimum dose required for many therapeutic agents among individuals is quite variable. Anatomical and physiological properties have a great influence on the pharmacokinetics of drugs and lead to inter- and intra-individual variability in the pharmacokinetics outcome [3]. Both inter- and intrasubject pharmacokinetic variability may be important. Intersubject variability is fundamental to the argument for using therapeutic drug monitoring. Intrasubject variability, due to the continually changing clinical state and pharmacokinetics observed in critically ill patients, has an impact on the ability to accurately predict a regimen.
A wide interindividual pharmacokinetic variability was described in clinically relevant populations such as pediatrics and critically ill patients. Growth and development are two specific features of children that are not observed in adults. And critically ill patients have a much higher level of sickness severity that is associated with profound pathophysiological changes. These particular features could lead to difficulties to attain therapeutic targets.

One well-known approach to characterize variability in pharmacokinetic parameters is nonlinear mixed effects modeling. It is a common approach to identify unexplained population variability in parameters of pharmacokinetic models and to identify covariates, which explain the variability of the data. Population models can then be developed using bayesian logistics to

Corresponding Author: Amélie Marsot, Faculté de Pharmacie, Pavillon Jean Coutu, 2940 chemin de Polytechnique, Montréal, E-Mail :

amelie.marsot@umontreal.ca 
obtain an estimation of the pharmacokinetic parameters of a given patient and predict the most adapted dose in light of the therapeutic target.

The aim of this article was to present the main covariates highlighted by pharmacokinetic modeling to explain the pharmacokinetic variability that exist in two specific populations, pediatrics and critically ill adults' patients. These covariates, explaining a part of the pharmacokinetic variability, allow subsequently to individualize treatment and to optimize drug dosing regimens.

\section{Pharmacokinetic modeling}

Models are mathematical tools used to describe real-world features. Models are usually simplified representations of systems; the nature of simplification is related to the intended use of the model. Neither is a "true" model but each may be fit for its intended purpose. Population modeling is a tool to identify and describe relationships between a subject's physiologic characteristics and observed drug exposure or response. Population pharmacokinetic modeling is not a new concept; it was first introduced in 1972 by Sheiner et al [4]. It was soon expanded to include models linking drug concentration to response (pharmacodynamics), thereafter modeling has grown to become an important tool in drug development and clinical practice.

Pharmacokinetics has been defined as "how the body handles the drug" and describes the relationship between the administered dose of drug and its concentration in the plasma or at its target site [5]. Pharmacokinetics models help identify sources of variability in the dose-concentration relationship of a drug and have been the focus of considerable attention because they are vital for linking pharmacokinetics information to measures of activity and clinical outcomes [6]. Pharmacokinetic models have usually several components: (i) structural models that describe the time course of a measured response, (ii) statistical models that describe the variability or random effects in the observed data and (iii) covariate models that describe the influence of factors such as demographics or disease on the individual time course of the response. The structural model is analogous to a systemic model (describing kinetics after IV dosing) and an absorption model (describing the drug uptake into the blood for extravascular dosing). When data are available from only a single site in the body (venous plasma for example), concentrations usually show 1,2 , or 3 exponential phases which can be represented using a systemic model with one, two, or three compartments, respectively. Insight into the appropriate compartment numbers can be gained by plotting log concentration vs. time. Each distinct linear phase when log concentrations are declining will generally need its own compartment. The statistical model describes variability around the structural model. There are two primary sources of variability in any population pharmacokinetic model: between-subject variability, which is the variance of a parameter across individuals; and residual variability, which is unexplained variability after controlling for other sources of variability. Some databases support estimation of between-occasion variability, where a drug is administered on two or more occasions in each subject that might be separated by a sufficient interval for the underlying kinetics to vary between occasions. Developing an appropriate statistical model is important for covariate evaluations and to determine the amount of remaining variability in the data. Identification of covariates that are predictive of pharmacokinetic variability is important in population pharmacokinetic evaluations. Identifying covariates that have an effect on pharmacokinetics can help to explain interindividual variability and thus improve the predictive value of the dosing model, thereby increasing the effectiveness of the dose-tailoring procedure. These different elements lead to estimation of pharmacokinetic parameters that can explain why a drug may display a different concentration-time profile in one patient group versus another.

\section{Specific pharmacokinetic aspects}

In Pediatric population

Historically, children were originally treated as 'small adults', i.e. the dose was simply scaled down per linear weight, leading potentially to overdosing in very small children, especially neonates, as their kidneys and liver were not yet fully developed, often resulting in slower drug elimination. Growth and development are two features of children that are not observed in adults [7]. Children, especially infants, are smaller, weigh less, and have a higher proportion of total body water and lower proportion of body fat compared with adults. Furthermore, key organ function, specifically kidney and liver function, is immature in newborns and infants. All the steps of pharmacokinetic (absorption, distribution, metabolism and elimination) are concerned. Firstly absorption, gastric $\mathrm{pH}$ is increased in neonates, infants, and young children and reaches adult $\mathrm{pH}$ values around 2 years old. Gastrointestinal motility 
is decreased in neonates and reaches adults levels in infants. Then, drugs are distributed to various body compartments according to its physicochemical properties. In neonates and infants, the total body water increased which contributes to an increase in the volume of distribution for hydrophilic drugs. For metabolism, enzymatic activity of metabolic enzymes, as CYP or uridine diphosphate glucuronosyltransferase (UGT) families, depends on genetic, physiologic and environmental factors [7].For example, expression of CYP1A2 at birth is negligible reaching $50 \%$ of adult expression by 0.9 years old, activity of CYP2C9 is close to $20 \%$ of adult value at birth and reaches $50 \%$ by 1 month of age, CYP2C19 activity is approximately $30 \%$ of adult activity at birth and achieved by 1 year old and activity of UGTs is deficient at birth and reaches adult levels at 2-4 years old [7]. Concerning elimination and renal clearance, the glomerular filtration rate increases steadily to $50-75 \%$ of adult function by 6 months and tubular secretion lags behind maturation of glomerular filtration by 7 months- 1 year. Renal function is fully mature by around 1 year old. Also, drug dynamics including desired and undesired side effects may be very different in newborn as the amplitude and the nature of the response may be different as compared to adults. Diseases states may also be different in newborn, compared with infants or adults, some of them being only observed in newborns. In addition, neonates and young infants may suffer from permanent effects resulting from stimulus applied at a sensitive point in development [8].

As awareness of developmental pharmacology subsequently expanded, the physiological differences in drug handling between children and adults were emphasized, leading to the notion that 'children are not small adults' [9]. However, rather than dichotomizing adult and pediatric patients, recognition that maturation is a continuous process has since led to acknowledgment of the need to quantify differences and understand similarities across the age range with appropriate scaling. These numerous physiological differences between adults and children, particularly infants, contribute to PK processes, and consequent ageand size-related differences in pharmacokinetic parameters [10]. Therefore, pharmacokinetic parameters, as doses, cannot simply be scaled linearly from adults to children. Almost 70 years ago, Crawford et al. noted that using body surface area is preferred over linear weight for predicting doses [11]. Decades later, it was suggested that a so-called allometric approach, which scales metabolic processes with body size, could also be used to explain changes in drug clearance [12]: $y_{\mathrm{i}}$ $=\mathrm{a} \mathrm{WT}_{\mathrm{i}}^{\mathrm{b}}$ where $\mathrm{y}_{\mathrm{i}}$ is the individual subject's body function of interest (that is being predicted), WT is the individual's body weight in kilograms, $a$ is the allometric constant, which assumes the value of $y$ when $\mathrm{WT}=1 \mathrm{~kg}$, and $\mathrm{b}$ is the allometric exponent $(b<1$ indicates that the body function increases slower with body size than weight). Using allometric models alone, which only account for size-related clearance changes, is not sufficient [13], particularly for neonates and infants, since clearance is frequently lower than expected in these pediatric populations due to the physiological immaturity of their organs. Therefore, age also needs to be taken into account, especially when analyzing data from neonates as their organ functions change very rapidly [10]. Taking both size and age into account in model development can help capture clearance changes across the whole pediatric age range.

\section{In critically ill patients}

Critically ill patients, hospitalized in intensive care unit (ICU) are markedly different from those in general ward environments and have significantly higher mortality rates. These patients have a much higher level of sickness severity that is associated with profound pathophysiological changes. These several pathophysiological changes can affect drug pharmacokinetic behavior [14]. Cardiovascular, renal, pulmonary and hepatic systems can be affected by critical illness. Many dysfunctions of the cardiovascular system could be at the origin of pharmacokinetic modifications, especially "third spacing" (i.e. a significantly increased interstitial volume, due to the administration of large volumes of resuscitation fluids in response to the resulting hypotension) [15]. Concerning the renal system, many of the commonly used drugs (such as antibiotics) in critically ill patients are subject to renal clearance and therefore alterations in renal function will affect concentrations of those drugs. As regards the pulmonary system, pneumonia is the most common infection in critically ill patients and is an important cause of morbidity and mortality in ICU patients [16]. Finally, hepatic dysfunctions can also affect critically ill patients, these dysfunctions may cause a decrease in drug metabolism and clearance.

These pathophysiological changes, leading to pharmacokinetic modifications could be included in the pharmacokinetic modelling.

Sources of pharmacokinetic variability In Pediatric population 
A number of covariates have been identified and use of these covariates improves prediction of the time-concentration profile in the individual child. Size, age, and organ function have been identified as three major covariates in pediatric population [17]. Marsot et al. identified also these significant covariates in their recent review (body weight, age and renal function) [18] (Figure 1).

Size is the most common covariate used to determine dose in pediatric population. The change in body weight with age is significant up to 1 year, body weight increases approximately three- to fourfold from birth to 1 year [19]. Although total body weight is used commonly, it is now more widely recognized that there is a nonlinear relationship between weight and drug elimination [20]. Allometric size modelling is used with increasing frequency in pediatric pharmacokinetic population analyses. It now widely recognised that there is a nonlinear relationship between weight and drug elimination capacity. It is possible to show that the log of the basal metabolic rate plotted against the $\log$ of the body weight produces a straight line with a slope of 0.75 . Allometric scaling also allows the direct comparison of paediatric estimates with adults when a weight standard of $70 \mathrm{~kg}$ is used. Nevertheless, body weight is insufficient to predict clearance in neonates and infants from adult estimates.

Age is the second covariate most used. Indeed, the first few years of life are time of growth and maturation of enzymatic processes. The addition of a model describing maturation is required, sigmoid hyperbolic or Hill model has been found useful for describing this maturation process but this model are little used [20]. Maturation of clearance begins before birth, suggesting that covariates like postmenstrual age (PMA) or gestational age (GA) would be better predictor of drug elimination than postnatal age (PNA). Indeed, the impact of ontogeny on the expression and functional activity of the major drug-metabolizing enzymes may be important. Whatever the definition of age (PCA or PMA, GA or PNA), this factor largely contributes to variability of drugs given to neonates and young infants but this impact will depend on the speed of maturation and the subpopulation studied.

The third covariate is organ function. Compromised hepatic or renal function alters the ability of these organs to clear drugs from the body. Indeed, renal function often estimated by serum creatinine ( $\mathrm{SCr}$ or $\mathrm{Cr}$ ), creatinine clearance (CLcr) or glomerular filtration rate (GFR) appears to be the third covariate in the review of Marsot et al. [18]. But this covariate is of interest only if the studied drug is excreted renally.

Others covariates such as pharmacogenetics and drug interaction seem to be interesting but they are less used. Indeed pharmacogenetic differences, such as single nuclear polymorphisms, may have dramatic impact on drug action. Phenotypic expression of genotypic differences may vary with age. This is particularly true of neonates and infants because enzyme systems are still maturing [21].

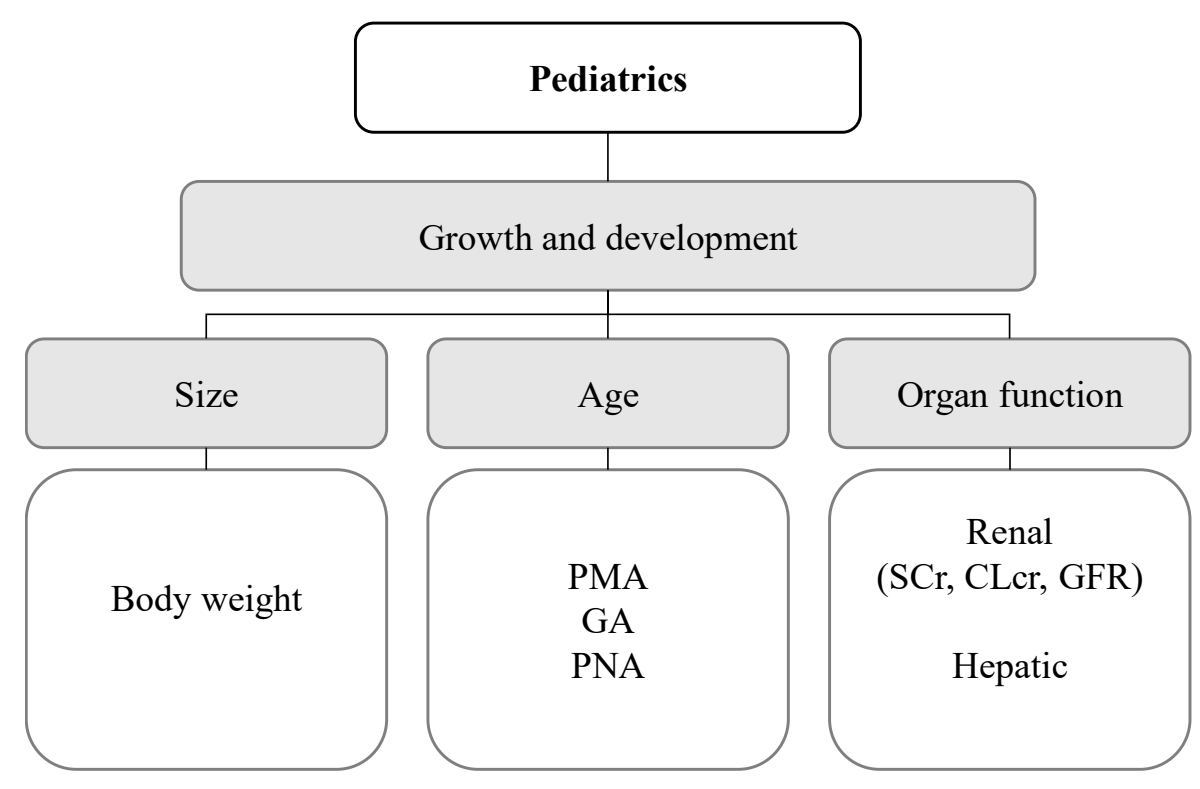

Figure 1. Main sources of pharmacokinetic variability in pediatrics

PMA: post menstrual age, GA: gestationnal age, PNA: post natal age, SCr: serum creatinine, CLcr: creatinine clearance, GFR: glomerular filtration rate 
In critically ill patients

Pharmacokinetic modeling allowed identifying relevant factors related to clinical outcome and to characterize and quantify their impact. Indeed, the main pharmacokinetic parameters (clearance and volume of distribution) were modified in this specific population. Numerous covariates have been included in drug modelling in critically ill patients (Figure 2).

Body weight, as for pediatric population, was an important covariate. The relationship between total body weight and the volume of distribution was often described and administered drug doses are commonly adjusted to total body weight. Nevertheless it is difficult to list main covariates in this population as it is done in pediatrics. Indeed, these patients have pathophysiological characteristics that vary widely and therefore many covariates associated. These covariates are included or not in the pharmacokinetic models developed based on the characteristics of the studied drug to explain the changes in the volume of distribution and clearance mainly.

Changes in the volume of distribution in critically ill patients result from critical illness related pathophysiology and consequent medical interventions such as fluid resuscitation. Modifications of cardiac and interstiatial fluids shifts result in a modification of volume of distribution, which may modify plasma drug levels [22]. Specifically, the increase in the volume of distribution in septic patients has been attributed to hypoalbuminaemia and the resultant decrease in oncotic pressure, which may progress with a shift in body water from the intervascular to the extravascular space [23]. Extracorporeal circuits, such as extracorporeal membrane oxygenation (ECMO) and renal replacement therapy (RRT) alter also the volume of distribution. Nevertheless, variability in the techniques used for RRT and ECMO is a significant limitation in the generalisability of models described with these covariates. Indeed, there is no standardized approach to delivering RRT, except for intermittent haemodialysis, drugs clearance can be highly variable across different RRT modalities and settings.

Clearance of drugs may be due to metabolism and/or elimination. Renal elimination is particularly affected in critically ill patients. A reduction in glomerular filtration rate, as occurs in acute kidney injury, reduces clearance of renally cleared drugs [24]. In contrast, augmented renal clearance may occur with some antibiotics as a result of increased renal perfusion caused due to high cardiac output and an associated enhanced blood flow to major organs [25]. As for volume of distribution, RRT has an impact on clearance mechanism and marked variability in performance of clearance by renal replacement therapy has been also described. Hepatic dysfunction can also affect elimination of drugs, which are metabolized by the liver or undergo transintestinal clearance, but little data is available for this population.

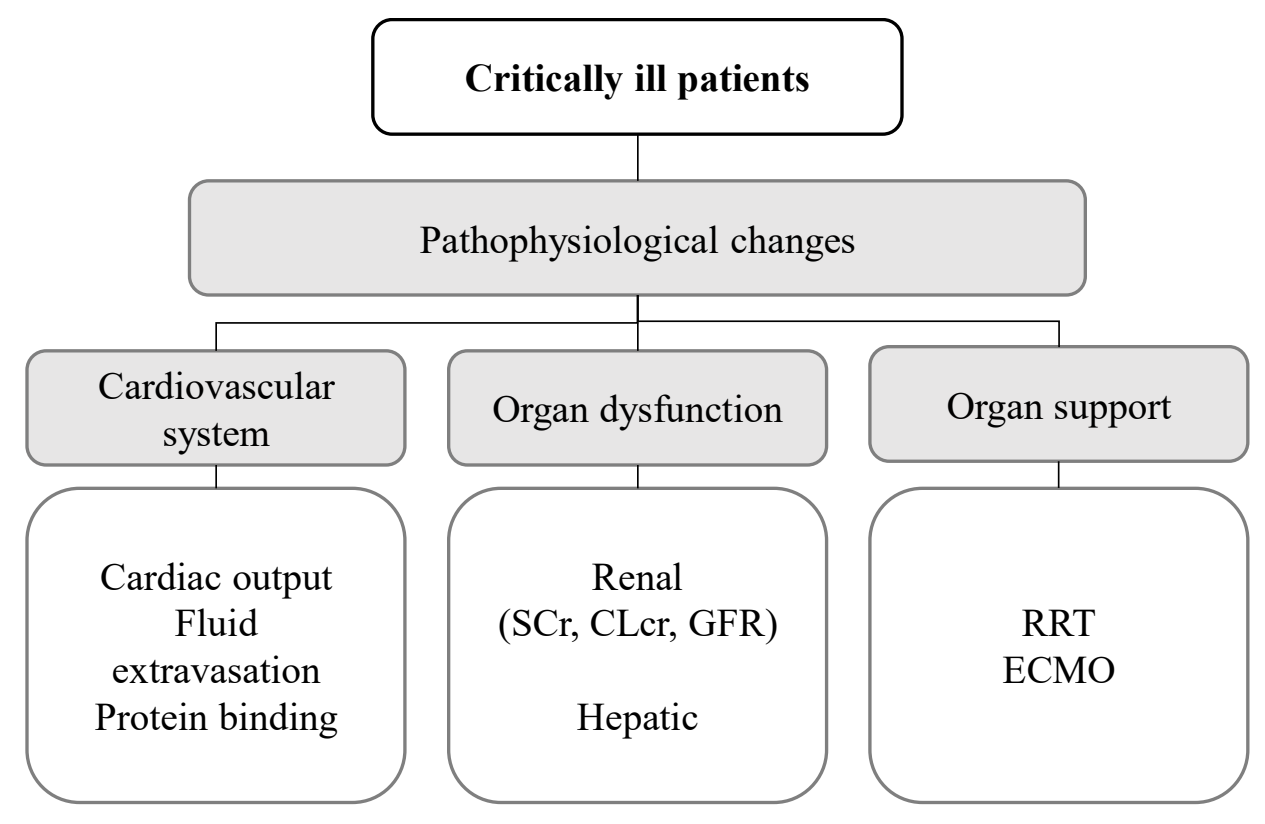

Figure 2. Main sources of pharmacokinetic variability in critically ill patients $S C r$ : serum creatinine, CLcr : creatinine clearance, GFR: glomerular filtration rate, RRT: renal replacement therapy, ECMO: extracorporeal membrane oxygenation 


\section{Applications in clinical practice \\ General}

Population pharmacokinetic models have various clinical applications after the drug becomes available to the market. The main clinical applications can be the therapeutic drug monitoring (26). Concerning the therapeutic drug monitoring, it is the measurement of drug concentrations to optimize dosing regimens for individual patients with the objective to maximize efficacy and minimize toxicity therapeutic drug monitoring can be applied by evaluating whether the drug concentrations are in the therapeutic range, but in case of deviating concentrations, or when the therapeutic target is not just a concentration but for example an AUC, it is difficult to provide a dosing recommendation manually. A more robust approach to individualize dosing by therapeutic drug monitoring is the maximum a posteriori probability Bayesian fitting procedure [27], which is implemented in various therapeutic drug monitoring software programs. Several therapeutic drug monitoring computer tools are available. In 2012, a review of 12 software tools was published [28]; the authors of the review recommend that most therapeutic drug monitoring software tools can be improved, more specifically the interface, user friendliness, data storage capability and report generation. An important aspect of reliable therapeutic drug monitoring programs is the choice of a population pharmacokinetic model, which must be suitable for the patient population for which therapeutic drug monitoring is performed. Neef et al. presented a case of vancomycin maximum a posteriori probability Bayesian adjustment where four different population pharmacokinetic models resulted in four strikingly different dosing schemes recommendations [29]. Another study evaluated different population pharmacokinetic models for amikacin and also showed significant differences in model performance [30].

\section{Example of vancomycin in pediatric population} Vancomycin remains a primary therapeutic choice against gram-positive pathogens in newborns and infants. There has been continued interest in describing vancomycin pharmacokinetics in this population for nearly 20 years. A review describing all population pharmacokinetic models of vancomycin was published by Marsot et al. in 2012. This review allows us to identify some covariates having an effect on interindividual variability and on the final values of the estimated pharmacokinetic parameters. Indeed, to optimize vancomycin dosage, this review point out the relevant covariates according to the target population. In pediatric population, the dosage optimization depends on age, body weight and $\mathrm{CL}_{\mathrm{cr}}$ as described previously. The assessment report on vancomycin drug products issued in 2017 by the European Medicines Agency is in line with the recommendations by Marsot et al. (31).

To evaluate the clinical utility and safety of a model-based patient-tailored dose of vancomycin in pediatrics, Leroux et al. developed a modelbased vancomycin dosing calculator from a population pharmacokinetic study that has been integrated into the routine clinical care in 3 neonatal intensive care units between 2012 and 2014 [32]. Individual clearance and volume of distribution were calculated based on the model including body weight (current and birth weight), PNA and serum creatinine, then loading and maintenance doses were calculated with these individual pharmacokinetic parameters. This study showed that the target attainment rate increased from $41 \%$ to $72 \%$ without any case of vancomycinrelated nephrotoxicity [32]. This model-based patient-tailored dose of vancomycin demonstrated positive results in terms of pharmacokinetics and safety. In conclusion, this study provides a proofof-concept for the clinical utility and safety of individualized dosing regimens based on population pharmacokinetic model.

\section{Example of amikacin in critically ill patients}

Amikacin is one of the most used antibiotics for the treatment of severe infections. There has been continued interest in describing amikacin pharmacokinetics for nearly 20 years, and several population pharmacokinetic models have been developed for patients with altered pharmacokinetic behaviour, including critically ill patients [33].

Roberts et al. described challenges and potential solutions to individualized antibiotic dosing for critically ill patients [34]. Different solutions were proposed with therapeutic drug monitoring, dosing nomograms and Bayesian dose adaptation. Bayesian dose adaptation appears to be the gold standard with many available programs to calculate individualized antibiotic doses for patients [28]. As for the developed model-based vancomycin dosing calculator in pediatrics, the dose optimization software uses the equations of pharmacokinetic model and the population Bayesian prior, together with the individual patient's observed drug concentrations to calculate a Bayesian posterior parameter value distribution for that patient. The dose optimization software then calculates the drug dose that minimizes the 
expected weighted squared error between the predicted and user-specified target drug concentrations. Many of these programs have or will have electronic medical record interfaces and smart-phone applications that can be used at the patient's bedside. With these softwares, the clinicians could enter the concentration-time data from therapeutic drug monitoring and patient's covariates data into software that personalizes a dosing regimen for patient to achieve an evidencebased pharmacokinetic (and pharmacodynamic) target.

\section{Pharmacokinetic modeling, variability and individualized dosing}

Therapeutic drug monitoring is increasingly giving way to dosing drugs based on population-based pharmacokinetic parameters, even when pharmacokinetic values vary quite a bit in individual patients.

The promise of personalized medicine offers better individualized therapy and improved treatment for all patients. Population pharmacokinetic models for drugs in pediatric and critically ill adults' patients have been developed from many drugs. These population pharmacokinetic models allow to calculated patient-specific pharmacokinetic parameters. Indeed, in Bayesian dose adaptation, the dose of a drug is adjusted to ensure an individual patient's exposure meets pharmacokinetic targets (Figure $3)$.

Information about a specific patient's plasma drug concentrations and a population pharmacokinetic model (including variability) from the relevant population are included. Whilst the term 'Bayesian' can conjure notions of mathematical complexity, the underlying principles are quite straightforward. A dose can be estimated for a patient using the population model embedded in the Bayesian system. This is a mathematical description of data obtained from a previous drug trial(s) and is considered 'prior' information. It can be used to estimate a 'typical' drug effect (concentration or response) and inform users of the range of variability likely to be seen. What is required is more information about the patient - in this case in the form of serum concentrations that can be added to the Bayesian software. Bayesian statistics and forecasting allows a new 'individualised' concentration-time curve to be estimated using the patient's individual PK parameters. The fundamental principle of the Bayesian approach is to learn about the "weighted average' of some prior beliefs and observations. As more concentrations are obtained, the model can be further optimised, and dose estimations can be made based on this updated model. One of the most practical uses for these systems is the ability to simulate dosing strategies. A variety of dose strategies could be simulated and the most favourable chosen by the treating team.

In summary, the use of individualized therapy correlating each patient's personal response to specific drug concentrations, rather than assuming a drug is therapeutic within a desired range, can improve clinical outcomes if the correct biomarkers are selected for response.

\section{CONCLUSION}

Population pharmacokinetic analyses are increasingly recognized by European or US regulatory agencies as an aid to clinical decisionmaking. Pharmacokinetic parameters are of clinical value for determining dose in a typical individual. However, main pharmacokinetic parameters $(\mathrm{CL}, \mathrm{V})$ are associated with variability. Identifying the sources of this variability allows us to individualize drug dose.

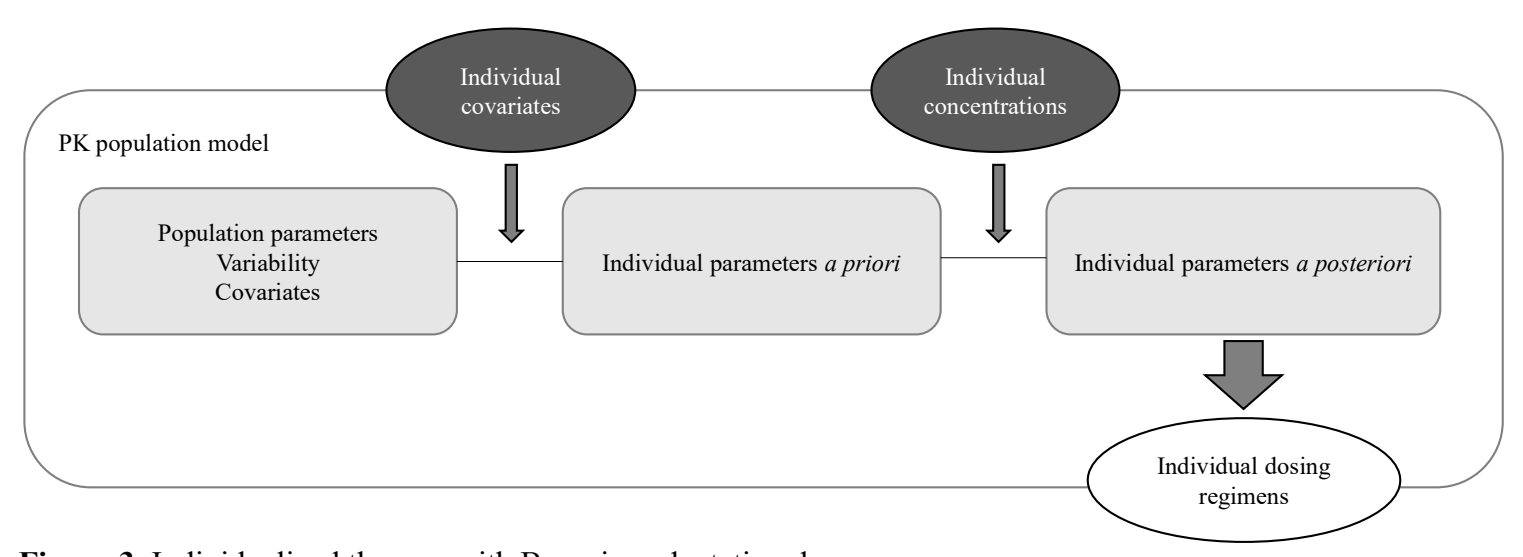

Figure 3. Individualized therapy with Bayesian adaptation dose PK: pharmacokinetic 
However, covariate-model-based dosing strategies have been translated in daily clinical practice for only a limited number of drugs. Nevertheless, covariate-based models have provided useful guidance for dose adjustments in many circumstances [32]. When used in routine practice, more-sophisticated Bayesian approaches to adaptive dosing have also been demonstrated to improve the efficacy-toxicity balance. This innovative personalized dosing approach is a promising way to optimize drug therapy in clinically relevant populations. Each individual is unique in this respect.

\section{REFERENCES}

1. Lu AYH. Drug-Metabolism Research Challenges in the New Millennium: Individual Variability in Drug Therapy and Drug Safety. Drug Metab Dispos. 1998 December 1; 26(12):1217-22.

2. Lippert J, Brosch M, von Kampen O, Meyer M, Siegmund HU, Schafmayer C, et al. A Mechanistic, Model-Based Approach to Safety Assessment in Clinical Development. CPT: pharmacomet syst pharmacol. 2012; 1:e13

3. Thummel KE, Lin YS. Sources of interindividual variability. Methods Mol Biol. 2014;1113:363-415

4. Sheiner LB, Rosenberg B, Melmon KL.Modelling of individual pharmacokinetics for computer-aided drug dosage. Comput Biomed Res. 1972 Oct;5(5):411-59

5. Rowland M, Tozer TN. Clinical pharmacokinetics/pharmacodynamics.

Philadelphia: Lippincott Williams and Wilkins, 2005

6. Holford NH, Sheiner LB. Understanding the doseeffect relationship: clinical application of pharmacokinetic-pharmacodynamic models. Clin Pharmacokinet. 1981 Nov-Dec;6(6):429-53

7. Anderson GD and Lynn AM. Optimizing pediatric dosing: A developmental pharmacologic approach. Pharmacotherapy 2009; 29(6): 680-90

8. Anderson BJ. My child is unique; the pharmacokinetics are universal. Paediatr Anaesth 2012; 22(6): 530-8.

9. Gillis J, Loughlan P. Not just small adults: the metaphors of paediatrics. Arch Dis Child. 2007;92(11):946-7

10. Kearns GL, Abdel-Rahman SM, Alander SW, Blowey DL, Leeder JS, Kauffman RE. Developmental pharmacology-drug disposition, action, and therapy in infants and children. N Engl J Med. 2003;349(12):1157-67.

11. Crawford JD, Terry ME, Rourke GM. Simplification of drug dosage calculation by application of the surface area principle. Pediatrics. 1950;5(5):783-90

12. Holford NH. A size standard for pharmacokinetics. Clin Pharmacokinet. 1996;30(5):329-32
13. Gilman JT. Therapeutic drug monitoring in the neonate and paediatric age group. Problems and clinical pharmacokinetic implications. Clin Pharmacokinet. 1990;19(1):1-10

14. Taccone FS, Laterre PF, Spapen H, Dugernier T, Delattre I, Layeux B, De Backer D, Wittebole X, Wallemacq P, Vincent JL and Jacobs F. Revisiting the loading dose of amikacin for patients with severe sepsis and septic shock. Crit Care. 2010;14(2):R53

15. van der Poll T. Immunotherapy of sepsis. Lancet Infect Dis. 2001 Oct;1(3):165-74. Review.

16. Vincent JL, Rello J, Marshall J, Silva E, Anzueto A, Martin CD, Moreno R, Lipman J, Gomersall C, Sakr Y, Reinhart K; EPIC II Group of Investigators. International study of the prevalence and outcomes of infection in intensive care units. JAMA. 2009 Dec 2;302(21):2323-9. doi: 10.1001/jama.2009.1754.

17. Tod M, Jullien V, Pons G. Facilitation of drug evaluation in children by population methods and modelling. Clin Pharmacokinet 2008; 47: 231-243.

18. Marsot A, Boulamery A, Bruguerolle B, Simon N. Population pharmacokinetic analysis during the first two years of life: an overview. Clinical Pharmacokinetics 2012; 51(12):787-98

19. Kearns GL and Reed MD. Clinical pharmacokinetics in infants and children. A reappraisal. Clin Pharmacokinet. 1989; 17: 29-67

20. Anderson BJ, Holford NH. Mechanistic basis of using body size and maturation to predict clearance in humans. Drug Metab Pharmacokinet 2009; 24(1): $25-36$

21. Neville KA, Becker ML, Goldman JL et al. Developmental pharmacogenomics. Pediatr Anesth 2011; 21: 255-265

22. Van Dalen R, Vree TB. Pharmacokinetics of antibiotics in critically ill patients. Intensive Care Med. 1990; 16(3):S235-8

23. Oparaoji EC, Cornwell EE $3^{\text {rd }}$, Hekmat E, Lum Cheong R, Adir JS and Siram S. Aminoglycoside volume of distribution in postoperative patients with septic shock. Clin Pharm. 1993; 12(2):131134

24. Felton TW, Hope WW, Roberts JA. How severe is antibiotic pharmacokinetic variability in critically ill patients and what can be done about it ? Diagnostic Microbiology and Infectious Disease 2014; 79:441-447

25. Di Giantomasso D, May CN, BellomoR. Vital organ blood flow during hyperdynamic sepsis. Chest. 2003; 124(3):1053-9

26. de Velde F, Mouton JW, de Winter BCM, van Gelder T, Koch BCP. Clinical applications of population pharmacokinetic models of antibiotics: Challenges and perspectives. Pharmacol Res. 2018 Aug; 134:280-288

27. R.W. Jelliffe, A. Schumitzky, D. Bayard, M. Milman, M. Van Guilder, X. Wang, et al., Modelbased, goal-oriented, individualised drug therapy. Linkage of population modelling, new' multiple 
model' dosage design, bayesian feedback and individualised target goals, Clin. Pharmacokinet. 1998;34 (1): 57-77

28. A. Fuchs, C. Csajka, Y. Thoma, T. Buclin, N. Widmer, Benchmarking therapeutic drug monitoring software: a review of available computer tools, Clin. Pharmacokinet. 2013;52 (1):9-22

29. C. Neef, D.J. Touw, A.R. Harteveld, J.J. Eerland, D.R. Uges, Pitfalls in TDM of antibiotic drugs: analytical and modelling issues, Ther. Drug Monit.2006; 28 (5) :686-689

30. M.D. Mar Fernandez de Gatta, M. Victoria Calvo, R. Ardanuy, A. Dominguez-Gil, J.M. Lanao, S.R. Moreno, Evaluation of population pharmacokinetic models for amikacin dosage individualization in critically ill patients, J. Pharm. Pharmacol. 2009;61 (6) :759-766

31. Assessment report: Vancomycin containing medicinal products (EMEA/H/A-31/1440) Committee for Medcinal Products for Human Use (European Medicines Agency) 2017

32. Leroux S, Jacqz-Aigrain E, Biran V, Lopez E, Madeleneau D, Wallon C, Zana-Taïeb E, Virlouvet AL, Rioualen S, Zhao W. Clinical Utility and Safety of a Model-Based Patient-Tailored Dose of Vancomycin in Neonates. Antimicrob Agents Chemother. 2016 Mar 25;60(4):2039-42. doi: 10.1128/AAC.02214-15. Print 2016 Apr

33. Marsot A, Guilhaumou R, Riff C, Blin O. Amikacin in critically ill patients: A review from population pharmacokinetics analysis. Clinical Pharmacokinetics 2017 Feb; 56(2):127-138. doi:10.1007/s40262-016-0428-X

34. Tsai D, Lipman J, Roberts JA. Pharmacokinetic/pharmacodynamic considerations for the optimization of antimicrobial delivery in the critically ill. Curr Opin Crit Care. 2015 Oct;21(5):412-20. doi: 10.1097/MCC.0000000000000229. Review. 